OPEN ACCESS

Edited by:

Ignazio Licata,

Institute for Scientific Methodology

(ISEM), Italy

Reviewed by:

Q. H. Liu,

Hunan University, China

Alberto Molgado,

Universidad Autónoma de San Luis

Potosi, Mexico

${ }^{*}$ Correspondence:

Tomas Veloz

tveloz@gmail.com

Specialty section:

This article was submitted to Mathematical Physics,

a section of the journal

Frontiers in Physics

Received: 06 August 2018

Accepted: 08 October 2018

Published: 13 November 2018

Citation:

Belmonte $F$ and Veloz T (2018) On the

Classical-Quantum Relation of

Constants of Motion.

Front. Phys. 6:121.

doi: 10.3389/fphy.2018.00121

\section{On the Classical-Quantum Relation of Constants of Motion}

\author{
Fabian Belmonte ${ }^{1}$ and Tomas Veloz ${ }^{2,3,4 *}$ \\ ${ }^{1}$ Department of Mathematics, Universidad Católica del Norte, Antofagasta, Chile, ${ }^{2}$ Institute of Philosophy and Complexity \\ Sciences, Santiago, Chile, ${ }^{3}$ Vrije Universiteit Brussel, Brussels, Belgium, ${ }^{4}$ Departamento Ciencias Biologicas, Facultad \\ Ciencias de la Vida, Universidad Andres Bello, Santiago, Chile
}

Groenewold-Van Hove theorem suggest that is not always possible to transform classical observables into quantum observables (a process known as quantization) in a way that, for all Hamiltonians, the constants of motion are preserved. The latter is a strong shortcoming for the ultimate goal of quantization, as one would expect that the notion of "constants of motion" is independent of the chosen physical scheme. It has been recently developed an approach to quantization that instead of mapping every classical observable into a quantum observable, it focuses on mapping the constants of motion themselves. In this article we will discuss the relations between classical and quantum theory under the light of this new form of quantization. In particular, we will examine the mapping of a class of operators that generalizes angular momentum where quantization satisfies the usual desirable properties.

\section{AMS subject classifications. 68Q25, 68R10, 68U05}

Keywords: example, LaTeX, quantization, constants of motion, reduction, diagonalization, angular momenta

\section{INTRODUCTION}

One of the deepest problems in the description of microscopic systems is the relation between their classical and quantum descriptions. Although there is no doubt in that quantum and classical descriptions perform well in their own scales of application, one would expect that a smooth transition between these two descriptions, at least at a theoretical level, is possible. However, it is well-known that this is not a trivial matter [1]. An area known as quantization has been intensely developed to understand such transition [2,3]. Quantization is thus a process that converts the mathematical description of a classical physical system into a description of a quantum system which resembles the classical system at a structural level. The aim of quantization goes beyond pure description of the transition between classical and quantum. Indeed, the process of quantization is not only the fundamental method used to develop important theories in physics such as particle and nuclear physics, quantum optics, among others [4], but also it is of fundamental importance in the philosophy of physics [5] .

Formally, a (canonical) quantization of a Poisson algebra $\mathcal{P}$ on a Hilbert space $\mathcal{H}$ is a family of linear and injective maps $\mathcal{O} p_{\hbar}^{\mathcal{P}}$ that associate to certain elements of $\mathcal{P}$ selfadjoint operators on $\mathcal{H}$ in a way that the quantum objects associated with the quantum observables (operators) correspond to the classical objects that represent the initial classical observable (elements of $\mathcal{P}$ ) in the limit $\hbar \rightarrow 0$. Weyl Calculus, which we shall denote by $\mathcal{O} p_{\hbar}$, is the main example of a quantization. It quantizes the canonical phase space $T^{*} \mathbb{R}^{n}=\mathbb{R}^{2 n}$. So in this case, $\mathcal{P}=C^{\infty}\left(\mathbb{R}^{2 n}\right)$ and $\mathcal{H}=L^{2}\left(\mathbb{R}^{n}\right)$. Weyl quantization was constructed so it additionally satisfies the identities $\mathcal{O} p_{\hbar}\left(x_{j}\right)=Q_{j}$ and $\mathcal{O} p_{\hbar}\left(\xi_{j}\right)=P_{j}$, where $x_{j}, \xi_{j}$ are the position and momentum coordinates respectively, and $Q_{j}, P_{j}$ are 
position and momentum operators respectively. Moreover, it settles their ordering by imposing selfadjointness of the operators corresponding to real valued functions on phase space (Weyl Calculus is the only Shubin's $\tau$-quantization satisfying that property). We must mention though, that there are various other frameworks developed to do quantization, such as strict deformation quantization [6], formal deformation quantization [7-9], or geometric quantization [3, 10]. In this article we are not going to consider them.

When Weyl ideas about quantization were first introduced, it was expected that quantization should hold the additional property of intertwining the Poisson bracket with the commutator, that is

$$
\mathcal{O} p_{\hbar}^{\mathcal{P}}(\{f, g\})=\frac{1}{\hbar}\left[\mathcal{O} p_{\hbar}^{\mathcal{P}}(f), \mathcal{O} p_{\hbar}^{\mathcal{P}}(g)\right]
$$

we call the latter a Dirac quantization. There is a famous result in the theory of quantization, first shown by Groenewold and later by van Howe, proving that there is no Dirac quantization for the canonical phase space satisfying the condition $\mathcal{O} p_{\hbar}^{\mathcal{P}}\left(x_{j}\right)=Q_{j}$ and $\mathcal{O} p_{\hbar}^{\mathcal{P}}\left(\xi_{j}\right)=P_{j}$; this result is known as Groenewold-van Howe no-go theorem (see $[1,11]$ or [12]). Although one would expect that Groenewold-van Howe no-go theorem is a unsurmountable problem for the theory of quantization, the existence of Dirac quantization would actually imply that, at least heuristically, classical mechanics is enough to describe quantum mechanics, and this would contradict fundamental results in quantum theory such as the Bell inequalities [13]. Along the latter line, it was shown that Weyl Calculus under general conditions satisfies that

$$
\mathcal{O} p_{\hbar}(\{f, g\})-\frac{1}{\hbar}\left[\mathcal{O} p_{\hbar}(f), \mathcal{O} p_{\hbar}(g)\right]=O(\hbar)
$$

This is an example of a semiclassical result.

For general phase spaces, the problem of finding a quantization is in general quite difficult. However, for the purposes of this article, we only need to consider the case when the phase space is the cotangent bundle of some Riemannian manifold $M$. In the latter case, different solutions can be found in the literature; we are going to work with the solution given by N. Landsman in his book [2], which satisfies many interesting properties. For instance, Landsman solution is equivalent to Weyl Calculus when $M=\mathbb{R}^{n}$.

In particular, we will focus on the relation between classical and quantum constants of motion (COM) in the quantization process. A constant of motion of a classical physical system is a quantity that is preserved during the system's evolution [14]. Although some COM correspond to intuitive properties of the system (e.g. energy), in many cases the COM of a system are not trivial [15]. A quantity $f$ of a classical physical system with a Hamiltonian $h_{0}$ is a COM if and only if $\left\{f, h_{0}\right\}=0$, where $\{\cdot, \cdot\}$ denotes the Poisson bracket. In order to establish the analogous definition of a COM for a quantum system, one considers a Hamiltonian $H_{0}$ and an observable $F$, and define $F$ to be a COM if and only if $\left[H_{0}, F\right]=0$, where $[\cdot, \cdot]$ is the quantum counterpart of the Poisson operator, known as commutator.
Groenwold-van Howe no-go theorem suggests that in general Weyl calculus does not preserves constants of motion for every $h_{0}$. Indeed, we can only conclude that

$$
\left\{f, h_{0}\right\}=0 \Rightarrow\left[\mathcal{O} p_{\hbar}(f), H_{0}\right]=O\left(\hbar^{2}\right) .
$$

Considering the analogies between the mathematical descriptions of classical and quantum COM, it seems natural that there should be a quantization process that preserves COM. In Belmonte [16], a solution for this problem was given. We shall recall its construction in section 2. We call this novel quantization a decomposable Weyl Calculus and we denote it by $\mathcal{O} p_{\hbar}^{d}$. Intuitively, one should think that the difference between the new (decomposable) and traditional Weyl calculus is that, instead of mapping position-momentum coordinates into position-momentum operators, decomposable Weyl Calculus satisfies a new constraint: mapping classical COM into quantum COM.

It is important to note that in some cases the right hand of (1) might be zero, in other words, it can happen that Weyl Calculus does preserve COM for some Hamiltonian $h_{0}$. The following theorem, proved in Belmonte [16], provides interesting examples when the equality holds.

THEOREM 1. Weyl calculus preserves constants of motion when $h_{0}$ is a function of the free Hamiltonian composed with a linear canonical transformation.

More precisely, if $\left\{h_{0}, f\right\}=0$ then $\left[H_{0}, \mathcal{O} p_{\hbar}(f)\right]=0$, whenever $h_{0}=\varphi \circ \tilde{h}_{0} \circ S$, where $\tilde{h}_{0}(x, \xi)=\|\xi\|^{2}, \varphi$ is a smooth regular function on $(0, \infty)$, and $S$ is a linear canonical transformation (i.e. a linear symplectomorphism). This result can be proved as well if we replace $h_{0}$ for the classical harmonic oscillator Hamiltonian, but we will not consider that case here.

As we will explain in section 2, every quantum COM admits a decomposition through the spectral diagonalization of $H_{0}$. In other words, if $\left[\mathrm{H}_{0}, \mathcal{O}_{\hbar}(f)\right]=0$, there is a field of operators $\left\{\operatorname{sp}(H) \ni \lambda \rightarrow\left[\mathcal{O} p_{\hbar}(f)\right]_{\lambda}\right\}$ that decomposes $\mathcal{O} p_{\hbar}(f)$. The latter motivates our work from the operator theory perspective, as it is important to compute explicitly $\left[\mathcal{O} p_{\hbar}(f)\right]_{\lambda}$.

The construction of the decomposable Weyl calculus, essentially, conjectures that $\left[\mathcal{O} p_{\hbar}(f)\right]_{\lambda}=\mathcal{O} p_{\hbar}^{\lambda}\left(f_{\lambda}\right)$, where $f_{\lambda}$ is the decomposition of $f$ in the reduced phase space at $\lambda$, and $\mathcal{O} p_{\hbar}^{\lambda}$ is a quantization for that space. This conjecture is equivalent to identity $\mathcal{O} p_{\hbar}(f)=\mathcal{O} p_{\hbar}^{d}(f)$; if this holds true we say that we have commutation of quantization and reduction on $f, \mathrm{CQR}$ for short.

An important COM in physics is angular momentum. Angular momentum is a constant of motion in any system where no external torque is acting. The conservation of momentum has been proven to correspond the rotational invariance by Noether's theorem [14]. In Belmonte [16], it was shown that every function on angular momenta is a constant of motion for $h_{0}(x, \xi)=$ $\|x\|^{2}$ or $h_{0}(x, \xi)=\|\xi\|^{2}$. Among those COM, an important role is played by the angular momenta coordinates themselves, i.e. the functions given by $l_{i j}(x, \xi)=x_{j} \xi_{i}-x_{i} \xi_{j}$. In particular, we have CQR on each $l_{i j}$. Thus, after quantizing, we obtain the operators $L_{i j}=i \hbar\left(x_{j} \partial_{i}-x_{i} \partial_{j}\right)$. Another important quantum COM 
is the operator $L^{2}:=\sum_{i<j} L_{i j}^{2}$. Even though the importance in quantum mechanics of $L^{2}$ is well known, the relation via quantization, of $L^{2}$ as a COM, with its classical counterpart has not been clarified. In this article we will study such relation, and thus our analysis adds up a novel example of CQR (using the well known quantum decomposition of $L^{2}$ ) to the already known cases [16]. Interestingly, the technique used to prove the main result (theorem 4) suggests that we should expect that we can obtain CQR for a broad class of COM.

It is well known that the spectral analysis of $L^{2}$ is based on the fact that we can restrict such analysis to one sphere, instead of working with the whole $\mathbb{R}^{n}$. The latter happens because the operators acting on each sphere obtained after decomposing $L^{2}$ through the diagonalization of $-\Delta$ are all unitarily equivalent. We will show that exactly the same can be done if we replace $L^{2}$ by any operator of the form $\mathcal{O} p_{\hbar}(f)$, where $f$ is a COM on which we have CQR and satisfying the additional condition $f\left(t x, t^{-1} \xi\right)=$ $f(x, \xi)$, for any $t>0$. In particular, this result applies to any function of angular momenta on which we have CQR. The additional condition seems artificial, but we will explain later why it emerges naturally.

This last result is an application of the decomposable Weyl calculus that provides an advantage over traditional Weyl calculus as one is able to calculate, even when CQR is not possible, the spectrum of the quantized operator directly from the quantization process.

The paper is organized as follows. In section 2 we will review the mathematical structure of COM in classical and quantum physics and introduce the decomposable Weyl calculus. In section 3 we provide examples of the decomposable Weyl calculus considering the free Hamiltonian and various functions of angular momenta. In section 4 , we develop the spectral analysis of $\mathcal{O} p_{\hbar}^{d}$ for the class of COM $f$ that satisfy $f\left(t x, t^{-1} \xi\right)=f(x, \xi)$. We conclude with a discussion of our results and future lines of work in section 5 .

\section{QUANTIZING THE CONSTANTS OF MOTION}

\subsection{Classical Constants of Motion}

In classical mechanics the phase space is described by a symplectic manifold $\Sigma^{2 n}$ (in our case $\Sigma=T^{*} \mathbb{R}^{n}=\mathbb{R}^{2 n}$ ), and observables are smooth functions in it. Denote by $\{\cdot, \cdot\}$ the corresponding Poisson bracket on $C^{\infty}(\Sigma)$. Fix a Hamiltonian $h_{0} \in C^{\infty}(\Sigma)$ and denote by $\Phi$ its flow. Then, for each regular $\lambda \in h_{0}(\Sigma)$, the energy level submanifold $\hat{\Sigma}_{\lambda}:=h_{0}^{-1}(\lambda) \subseteq \Sigma$ is invariant under $\Phi_{t}$. It turns out that the orbits space $\Sigma_{\lambda}$ : = $\hat{\Sigma}_{\lambda} / \Phi$ forms a symplectic manifold of dimension $2 n-2$ (this is a particular case of Marsden-Weinstein reduction or of the JacobiLiouville theorem, see [17]). We denote by $\pi_{\lambda}: \hat{\Sigma}_{\lambda} \rightarrow \Sigma_{\lambda}$ the canonical quotient map.

For example, in the case $h_{0}(x, \xi)=\|\xi\|^{2}$, it is easy to check that $\Phi_{t}(x, \xi)=(x-2 t \xi, \xi)$ and cleary $\hat{\Sigma}_{\lambda}=\mathbb{R}^{n} \times \mathbb{S}_{\sqrt{\lambda}}^{n-1}$. Moreover, $\Sigma_{\lambda}=T^{*} \mathbb{S}_{\sqrt{\lambda}}^{n-1}$ and the quotient map is given by $\pi_{\lambda}(x, \xi)=\left(\xi, x-\frac{\langle x, \xi\rangle}{\lambda} \xi\right)$, where we are identifying $T_{\xi}^{*} \mathbb{S}_{\sqrt{\lambda}}^{n-1}$ with the plane tangent to $\xi$ (literally). See Belmonte [16] for the proof of the last claim.

A classical observable $f \in C^{\infty}(\Sigma)$ is a constant of motion if $\left\{f, h_{0}\right\}=0$. Leibniz's rule and Jacobi identity show that the set $\mathcal{A}$ of all constants of motion form a Poisson subalgebra of $C^{\infty}(\Sigma)$. It is easy to show that $f \in \mathcal{A}$ if and only if $f \circ \Phi_{t}=f$, for each $t \in \mathbb{R}$. Thus, for each $f \in \mathcal{A}$, we can consider the field of functions $f_{\lambda} \in C^{\infty}\left(\Sigma_{\lambda}\right)$ given by

$$
f_{\lambda}\left(\pi_{\lambda}(\sigma)\right)=f(\sigma)
$$

where $\sigma \in \hat{\Sigma}_{\lambda}$. In particular, we can consider the flow $\Phi_{t}^{\lambda}[f]$ of $f_{\lambda}$ in $\Sigma_{\lambda}$. It is not difficult to show that $\Phi_{t}^{\lambda}[f] \circ \pi_{\lambda}=\pi_{\lambda} \circ \Phi_{t}[f]$, where $\Phi_{t}[f]$ is the flow of $f$ in $\Sigma$. This is a particular case of theorem 4.3.5 in [17]. For example, $h$ itself is a constant of motion and we have

$$
h_{\lambda} \equiv \lambda
$$

and

$$
\Phi_{t}^{\lambda}[h]=I d .
$$

\subsection{Quantum Constants of Motion}

In quantum mechanics the phase space is the projective space $\mathbb{P} \mathcal{H}$ of a Hilbert space $\mathcal{H}$ (in our case $\mathcal{H}=L^{2}\left(\mathbb{R}^{n}\right)$ ), and observables are selfadjoint operators acting on $\mathcal{H}$.

Let $H_{0}$ be a quantum Hamiltonian and $\mathrm{sp}\left(H_{0}\right)$ its spectrum. Then, there is a unique Borel measure $\eta$ (up to equivalence) on $\operatorname{sp}\left(H_{0}\right)$, a unique $\eta$-measurable field of Hilbert spaces $\left\{\operatorname{sp}\left(H_{0}\right) \ni\right.$ $\lambda \rightarrow \mathcal{H}(\lambda)\}$ (up to $\eta$ ), and a unique unitary operator $T: \mathcal{H} \rightarrow$ $\int_{\mathrm{sp}\left(H_{0}\right)}^{\oplus} \mathcal{H}(\lambda) d \eta(\lambda)$ such that

$$
\left[T H_{0} u\right](\lambda)=\lambda(T u)(\lambda) \forall u \in \operatorname{Dom}(H) .
$$

and

$$
\left[T e^{i t H_{0}} u\right](\lambda)=e^{i t \lambda}(T u)(\lambda) \forall u \in \mathcal{H}, t \in \mathbb{R} .
$$

$T$ is called the diagonalization of $H_{0}$. See [18] or [19] for details.

For example, in order to compute $T$ when $H_{0}=\Delta$, we introduce first

$$
T_{0}: L^{2}\left(\mathbb{R}^{n}\right) \rightarrow \int_{[0, \infty)}^{\oplus} L^{2}\left(\mathbb{S}_{\sqrt{\lambda}}^{n-1}\right) d \lambda
$$

given by

$$
T_{0} u(\lambda)=\left.2^{-\frac{1}{2}} \lambda^{-\frac{1}{4}} u\right|_{\mathbb{S}_{\sqrt{\lambda}}^{n-1}}
$$

where $\mathbb{S}_{\sqrt{\lambda}}^{n-1}$ is endowed with its canonical measure given by the metric induced from $\mathbb{R}^{n}$.

Note that co-area formula implies that $T_{0}$ is unitary. $T_{0}$ diagonalizes the operator $Q^{2}$, therefore $T=T_{0} \mathcal{F}$ diagonalizes $-\Delta$, where $\mathcal{F}$ is the Fourier transform. 
In the literature, $T_{0}$ (and therefore $T$ ) is usually presented in a slightly different way ([20], section 2$)$. First consider the unitary operators $S_{\lambda}: L^{2}\left(\mathbb{S}_{\sqrt{\lambda}}^{n-1}\right) \rightarrow L^{2}\left(\mathbb{S}^{n-1}\right)$ given by

$$
S_{\lambda} u(z)=\lambda^{\frac{n-1}{4}} u(\sqrt{\lambda} z)
$$

This unitary operators allow us to replace $\mathcal{H}(\lambda)=L^{2}\left(\mathbb{S}_{\sqrt{\lambda}}^{n-1}\right)$ on the direct integral above by $\mathcal{H}(\lambda)=L^{2}\left(\mathbb{S}^{n-1}\right)$. In this way, we obtain $T_{0}: L^{2}\left(\mathbb{R}^{n}\right) \rightarrow \int_{[0, \infty)}^{\oplus} L^{2}\left(\mathbb{S}^{n-1}\right) d \lambda$ and

$$
T_{0} u(\lambda, z)=2^{-\frac{1}{2}} \lambda^{\frac{n-2}{4}} u(\sqrt{\lambda} z)
$$

This form of $T_{0}$ is usually obtained using spherical coordinates directly. However, our version of $T_{0}$ is somehow more natural, because $Q^{2}$ restricted to $L^{2}\left(\mathbb{S}_{\sqrt{\lambda}}^{n-1}\right)$ coincides by definition with the constant scalar operator $\lambda$, which is exactly what we want to happen on $\mathcal{H}(\lambda)$. Therefore, the use of the unitary operators $S_{\lambda}$ to relate the two ways to obtain $T_{0}$ explained above play an important role in our construction, as we shall explain in detail in the remaining of the article.

A quantum observable, i.e. a selfadjoint operator $F$, is a quantum COM if $F$ strongly commutes with $H_{0}$. Notice that this is equivalent to $e^{i t H_{0}} F \subseteq F e^{i t H_{0}}$ [18].

$F$ is a constant of motion if and only if it admits a decomposition through $T[18,19]$, i.e. there is a measurable field of selfadjoint operators $\left\{\operatorname{sp}\left(H_{0}\right) \ni \lambda \rightarrow F_{\lambda}\right\}$ such that $[T F u](\lambda)=F_{\lambda}[T u(\lambda)]$. Such field of operators are the quantum counterpart of the field of classical observables $f_{\lambda}$. Just as in the classical context, we also have decomposition of the dynamics: clearly $e^{i t F}$ commutes with $H_{0}$ and we have that $\left[e^{i t F}\right]_{\lambda}=e^{i t F_{\lambda}}$.

Analogously to the classical case, the set $\mathfrak{A}$ of bounded quantum constants of motion is also an algebra (in fact, a von Neumann algebra). We also know that, if $F \in \mathfrak{A}$ then

$$
\|F\|=\sup _{\lambda}\left\|F_{\lambda}\right\|
$$

From the spectral theory point of view, it is important to find the field of operators decomposing a given quantum constant of motion $F$, since we know that

$$
\operatorname{sp}(F)=\overline{\cup_{\lambda} \operatorname{sp}\left(F_{\lambda}\right)}
$$

\subsection{The Decomposable Weyl Calculus}

Identities (2) and (3) share two crucial features. They reflect that the construction of the fibers (both classical and quantum) aim at, on the one hand, making constant the Hamiltonian on each fiber, and on the other hand, making trivial the corresponding dynamics on each fiber. These two common features point to an additional (and unexpected) shared feature: for both classical and quantum Hamiltonians, their COM can be decomposed through the respective fibers.

Therefore, we claim that $\mathcal{H}(\lambda)$ is the natural quantum counterpart of $\Sigma_{\lambda}$. So, if $H_{0}=\mathcal{O} p_{\hbar}\left(h_{0}\right)$ one could expect that we should be able to quantize $\Sigma_{\lambda}$ into $\mathcal{H}(\lambda)$. The main problem with this assertion is that in general we do not know what is the relation between $h_{0}(\Sigma)$ and $\operatorname{sp}\left(H_{0}\right)$, though in the semi-classical limit (under certain circumstances) $h_{0}(\Sigma)=\operatorname{sp}\left(H_{0}\right)$ [21, 22].

For example, when $h_{0}(x, \xi)=\|\xi\|^{2}$, we are able to quantize $\Sigma_{\lambda}$ into $\mathcal{H}(\lambda)$. Indeed, since $\Sigma_{\lambda}=T^{*} \mathbb{S}_{\sqrt{\lambda}}^{n-1}$ and $\mathcal{H}(\lambda)=$ $L^{2}\left(\mathbb{S}_{\sqrt{\lambda}}^{n-1}\right)$, we are able to transform smooth functions on $T^{*} \mathbb{S}_{\sqrt{\lambda}}^{n-1}$ into selfadjoint operators on $L^{2}\left(\mathbb{S}_{\sqrt{\lambda}}^{n-1}\right)$ by applying the WeylLandsman quantization [2], which we shall denote from now on by $\mathcal{O} p_{\hbar}^{\lambda}$.

In Belmonte [16] one of the authors built up the following quantization that by construction preserves constants of motion.

Definition 2. Assume that $\operatorname{sp}\left(H_{0}\right) \subseteq h_{0}(\Sigma)$. Let $\mathcal{A}^{d}$ be the space of functions $f \in \mathcal{A}$ such that $f_{\lambda}$ is an admissible symbol for $\mathcal{O} p_{\hbar}^{\lambda}$ for almost every $\lambda$. For each $f \in \mathcal{A}^{d}$, we define

$$
\mathcal{O} p_{\hbar}^{d}(f):=T^{*}\left[\int_{\operatorname{sp}\left(H_{0}\right)}^{\oplus} \mathcal{O} p_{\hbar}^{\lambda}\left(f_{\lambda}\right) d \eta(\lambda)\right] T,
$$

where $T^{*}$ is the adjoint operator of $T$ defined in (3).

We call $\mathcal{O} p_{\hbar}^{d}$ the ho-decomposable Weyl calculus and $\mathcal{A}^{d}$ the space of admissible symbols for $\mathcal{O}_{\hbar}^{d}$.

\section{Remark:}

The crucial reasons to introduce this novel quantization are

a) Contrary to Weyl calculus $\mathcal{O} p_{\hbar}$, the decomposable calculus $\mathcal{O} p_{\hbar}^{d}$ preserves constants of motion by construction.

b) When $\left[H_{0}, O p_{\hbar}(f)\right]=0$ for some $f \in \mathcal{A}$, the decomposable Weyl calculus construction provides the ansatz $\left[\mathcal{O} p_{\hbar}(f)\right]_{\lambda}=$ $\mathcal{O} p_{\hbar}^{\lambda}\left(f_{\lambda}\right)$ to compute the field of operators decomposing $\mathcal{O} p_{\hbar}(f)$.

Interestingly, when the ansatz in b) holds, we are able to compute the spectrum of $\mathcal{O} p_{\hbar}(f)$.

When $\left[\mathcal{O} p_{\hbar}(f)\right]_{\lambda}=\mathcal{O} p_{\hbar}^{\lambda}\left(f_{\lambda}\right)$, which is equivalent to satisfy $\mathcal{O} p_{\hbar}(f)=\mathcal{O} p_{\hbar}^{d}(f)$, we will say that we have a commutation of quantization and reduction, $\mathrm{CQR}$ for short, on $f$. Some examples of CQR are studied in Belmonte [16]. In the remaining of this article, we aim at giving a new example of CQR and at discussing how the ideas behind the involved proofs can lead to more general cases on which we have CQR on $f$.

\section{ANGULAR MOMENTUM IN THE FREE HAMILTONIAN}

Recall that we are interested in studying the case $h_{0}(x, \xi)=\|\xi\|^{2}$ on $\mathbb{R}^{2 n}$. However, we would like to note that using the method developed in Belmonte [16], we can consider as well the more general case $\tilde{h}_{0}=\varphi \circ h_{0} \circ S$, where $\varphi$ is a smooth regular function on $[0, \infty)$ and $S$ is a linear canonical transformation (symplectomorphism). An example of the latter is $\tilde{h}_{0}(x, \xi)=$ $\varphi\left(\|x\|^{2}\right)$.

Clearly, we have that $\mathcal{O} p_{\hbar}\left(h_{0}\right)=H_{0}=-\hbar^{2} \Delta$, where $\Delta$ is the Laplace operator. Moreover, since the flow of $h_{0}$ is linear and its metaplectic representation coincides with $e^{i t H_{0}}$, Weyl 
Calculus preserves COM under these conditions. In other words if $\left\{h_{0}, f\right\}=0$, then $\left[H_{0}, \mathcal{O} p_{\hbar}(f)\right]=0$ (see [16], for a detailed proof).

An important example of CQR is the following: Let $v$ be a complete vector field on $\mathbb{R}^{n}$ and define $f_{v}(x, \xi)=\langle v(x), \xi\rangle(v(x)$ should be interpreted as a vector on the tangent plane at $x$ and $\xi$ as a vector on the cotangent plane). It was proved in Belmonte [16] that, if $v$ is tangent to each sphere, then $f_{v}$ is a COM and we have CQR on $f_{v}$.

\subsection{Functions of Angular Momenta}

Consider the classical angular momenta functions

$$
l_{i, j}(x, \xi):=x_{j} \xi_{i}-x_{i} \xi_{j}
$$

Then any function of the form

$$
f(x, \xi)=\psi\left(l_{1,2}(x, \xi), \cdots, l_{n-1, n}(x, \xi)\right)
$$

is a COM, where $\psi \in C^{\infty}\left(\mathbb{R}^{\frac{n(n-1)}{2}}\right)$. We will see later that some of those functions have been already associated with differential operators in $L^{2}\left(\mathbb{S}_{\sqrt{\lambda}}^{n-1}\right)$, so they should form a class of COM where CQR is applicable.

Of course each $l_{i j}$ is a COM, in fact $l_{i j}=f_{v_{i j}}$, where $v_{i j}$ is the infinitesimal generator of the rotation of the plane generated by the elements $e_{i}$ and $e_{j}$ of the canonical base of $\mathbb{R}^{n}$. So, we have CQR on each $l_{i j}$. After applying $\mathcal{O} p_{\hbar}$ on each $l_{i j}$, we obtain the angular momenta operators $L_{i j}=i \hbar\left(x_{i} \partial_{j}-x_{j} \partial_{i}\right)$.

Another important example of a quantum constant of motion in our case is the operator $L^{2}:=\sum_{i<j} L_{i j}^{2}$. It will give us an important example of CQR. Its analysis is given in any basic course on quantum mechanics, but we shall recall it to explain how we obtain our case and why we expect that its spectral analysis can be performed for any operator of the form $\mathcal{O} p_{\hbar}(f)$, whenever $f$ is a classical constant of motion on which we have CQR.

\subsection{The Canonical Analysis of $L^{2}$}

The operator $L^{2}$ is of special interest in quantum theory not only because $L^{2}$ is a COM, but also for solving the free particle Schrödinger equation as

$$
\begin{aligned}
\Delta & =\frac{1}{r^{2}} \frac{\partial}{\partial r}\left(r^{2} \frac{\partial}{\partial r}\right)+\frac{1}{r^{2} \sin (\theta)} \frac{\partial}{\partial \theta}\left(\sin \theta \frac{\partial}{\partial \theta}\right)+\frac{1}{r^{2} \sin ^{2}(\theta)} \frac{\partial^{2}}{\partial \phi^{2}} \\
& =\frac{1}{r^{2}} \frac{\partial}{\partial r}\left(r^{2} \frac{\partial}{\partial r}\right)-\frac{L^{2}}{\hbar^{2} r^{2}}
\end{aligned}
$$

Therefore one is able to apply a separation of variables, and obtain that

$$
\begin{aligned}
& L^{2}|l, m\rangle=\hbar^{2} l(l+1)|l, m\rangle \\
& L_{z}|l, m\rangle=\hbar m|l, m\rangle
\end{aligned}
$$

with $\langle\theta, \phi \mid l, m\rangle=Y_{l, m}(\theta, \phi)$, where $Y_{l, m}(\theta, \phi)$ is the spherical harmonics with index $l$ (orbital angular momentum number) and $m$ (magnetic number). The latter analysis of $L^{2}$ can be applied to any Hamiltonian with a radial potential [23].

Equation (9) implies that the restriction of $L^{2}$ to each sphere coincides with the Laplace-Beltrami operator [24], page 164.

Interestingly, the fact that the separation of variables method is successful in the Schrödinger equation represented in spherical coordinates, and that such separation leaves the radial part of the equation independent of the angular part is intimately related to the fact that in the classical counterpart the fibers of the free Hamiltonian are cotangent bundles of spheres.

The canonical spectral analysis of $L^{2}$ involves its decompositions, but it is usually presented in a different way: Let $L_{\lambda}^{2}$ be the field of operators decomposing $L^{2}$. Since $L^{2}$ is invariant by Fourier transform, $L_{\lambda}^{2}$ is just the restriction of $L^{2}$ to the corresponding sphere. It is straightforward to check that $L_{\lambda}^{2}=S_{\lambda}^{*} L_{1}^{2} S_{\lambda}$, where $S_{\lambda}$ is given by (4). In particular, $\operatorname{sp}\left(L^{2}\right)=\operatorname{sp}\left(L_{1}^{2}\right)$. This is the reason why we are able to complete the spectral analysis of $L^{2}$ by calculating eigenvectors on the sphere (the spherical harmonics), but usually this is not mentioned in the literature.

Notice that the latter analysis applies to $L^{2}$ but might fail for other quantum constants of motion $F$. Namely, the operators $S_{\lambda}$ migh not implement a unitary equivalence between the operators decomposing $F$. However, in section 4 , we will show that such equivalence holds true for any operator of the form $\mathcal{O} p_{\hbar}(f)$, when $f$ is any function of angular momenta on which we have CQR.

Angular momentum poses an excellent case of study for the problem of quantization, as the commutation relations in the classical and quantum counterpart strongly resemble each other (for the canonical case $n=3$ ):

$$
\begin{aligned}
\left\{l_{i}, l_{j}\right\} & =\epsilon_{i j k} l_{k} \\
{\left[L_{i}, L_{j}\right] } & =i \hbar \sum_{k=1}^{3} \epsilon_{i j k} L_{k},
\end{aligned}
$$

with $i, j, k \in\{x, y, z\}$. Moreover, the commutation relations between the angular momentum operators and the total momentum operator are also preserved.

$$
\left\{l_{i}, l^{2}\right\}=\left[L_{i}, L^{2}\right]=0,
$$

The latter equalities said that $L^{2}$ is a Casimir element of the Lie algebra generated by the $L_{j}$ (actually, it is a Casimir element of the corresponding universal enveloping algebra); we will come back to this point later.

\subsection{The Decomposable Weyl Calculus on $L^{2}$}

The operator $L^{2}$ has a natural classical counterpart:

$$
l^{2}(x, \xi):=\sum_{i<j} l_{i j}^{2}(x, \xi)
$$

The reader might expect that $\mathcal{O} p_{\hbar}\left(l^{2}\right)=L^{2}$, but this is not true. The reasons why this happens are developed in the following results. 
Proposition 3. $O p_{\hbar}\left(l^{2}\right)=L^{2}+\frac{n(n-1)}{4} \hbar^{2} I$

Proof: Using the canonical commutation relations and the well known formula to obtain the value under $\mathcal{O}_{p_{\hbar}}$ of any polynomial ([12], page 86), we get that $\mathcal{O} p_{\hbar}\left(l_{i j}^{2}\right)=L_{i j}^{2}+\frac{1}{2} \hbar^{2} I$. Since there are $\frac{n(n-1)}{2}$ terms in the sum defining $l^{2}$, we obtain our result.

Obviously $l^{2}$ commutes with $h_{0}$, so it can be decomposed through each reduction. Recall that $\Sigma_{\lambda}=T^{*} \mathbb{S}_{\sqrt{\lambda}}^{n-1}$, therefore $l_{\lambda}^{2} \in C^{\infty}\left(T^{*} \mathbb{S}_{\sqrt{\lambda}}^{n-1}\right)$. The following is the central result of this article.

THEOREM 4.

$$
l_{\lambda}^{2}(q, p)=\lambda\left(g_{q}^{\lambda}\right)^{-1}(p, p)
$$

where $g_{q}^{\lambda}$ is the metric on $T_{q} \mathbb{S}_{\sqrt{\lambda}}^{n-1}$ induced from $\mathbb{R}^{n}$ and $\left(g_{q}^{\lambda}\right)^{-1}$ is the corresponding metric on $T_{q}^{*} \mathbb{S}_{\sqrt{\lambda}}^{n-1}$.

We will present two different proofs of this result. The first one is straightforward, while the second one is more sophisticated but relies on the underlying symmetry of the sphere, and we shall explain later why this reasoning is important for further developments.

Proof: Since the metric on $T_{q} \mathbb{S}_{\sqrt{\lambda}}^{n-1}$ is induced from $\mathbb{R}^{n}$, we have that $\left(g_{q}^{\lambda}\right)^{-1}(p, p)=\langle p, p\rangle=\|p\|^{2}$ (once we identify $T_{q} \mathbb{S}_{\sqrt{\lambda}}^{n-1}$ with the hyperplane tangent to $q$ on $\mathbb{R}^{n}$ ). Moreover, under the constraints $\|q\|^{2}=\lambda$ and $\langle q, p\rangle=0$, we have that

$$
\begin{aligned}
2 l^{2}(q, p)= & \sum_{i, j}\left(q_{i} p_{j}-q_{j} p_{i}\right)^{2}=\sum_{j} p_{j}^{2}\left(\sum_{i} q_{i}^{2}\right) \\
& -2 \sum_{i} q_{i} p_{i}\left(\sum_{j} q_{j} p_{j}\right)+\sum_{i} p_{i}^{2}\left(\sum_{j} q_{j}^{2}\right)=2 \lambda\|p\|^{2} .
\end{aligned}
$$

Our second proof relies on some results from Poisson geometry which we shall briefly explain. Let $J_{\lambda}: T^{*} \mathbb{S}_{\sqrt{\lambda}}^{n-1} \rightarrow$ $\mathfrak{s o}(n)^{*}$ the natural moment map, where $\mathfrak{s o}(n)^{*}$ is the dual of the Lie algebra $\mathfrak{s o}(n)$. It is well known that $J_{\lambda}$ is covariant, i.e. $J_{\lambda}[U(q, p)]=A d_{U}^{*}[J(q, p)]$, where $U \in S O(n)$ (the special orthogonal group), $A d^{*}$ is the coadjoint action, and $U(q, p)=$ $\left(U q,\left(U^{*}\right)^{-1} p\right)$. Let $v \in \mathfrak{s o}(n)$ and define $E_{v} \in C^{\infty}\left(\mathfrak{s o}(n)^{*}\right)$ by $E_{v}(z)=\langle v, z\rangle$. Also, let $v_{i j}$ the canonical base of $\mathfrak{s o}(n)$. It is easy to show that $J_{\lambda}^{*}\left(E_{v_{i j}}\right)=l_{i j}^{\lambda}$. Also notice that $C:=\sum E_{v_{i j}}^{2}$ is $A d^{*}$ invariant (in particular, it is a Casimir element of $C^{\infty}\left(\mathfrak{s o}(n)^{*}\right)$ ) and $l_{\lambda}^{2}=J_{\lambda}^{*} C$. In particular, $l_{\lambda}^{2}\left(U(q),\left(U^{*}\right)^{-1} p\right)=l_{\lambda}^{2}(q, p)$.

Proof: It is easy to show that the equality holds when $q=\sqrt{\lambda} e_{n}$, where $e_{n}$ is the last member of the canonical base (or any of them actually). Let $q \in \mathbb{S}_{\sqrt{\lambda}}^{n-1}$ and fix $U \in S O(n)$ such that $U(q)=\sqrt{\lambda} e_{n}$. Since the canonical action of the orthogonal group preserves the metric, we have that

$$
\lambda\left(g_{q}^{\lambda}\right)^{-1}(p, p)=\lambda\left(g_{\sqrt{\lambda} e_{n}}^{\lambda}\right)^{-1}\left(\left(U^{*}\right)^{-1} p,\left(U^{*}\right)^{-1} p\right)
$$

$$
=l_{\lambda}^{2}\left(U(q),\left(U^{*}\right)^{-1} p\right)=l_{\lambda}^{2}(q, p)
$$

and this finishes the proof.

COROLlaRY 5. $\mathcal{O} p_{\hbar}^{\lambda}\left(l_{\lambda}^{2}\right)=L_{\lambda}^{2}+\frac{n(n-1)}{4} \hbar^{2} I$, in other words $\left[\mathcal{O} p_{\hbar}\left(l^{2}\right)\right]_{\lambda}=\mathcal{O} p_{\hbar}^{\lambda}\left(l_{\lambda}^{2}\right)$

Proof: Since the Ricci scalar (or scalar curvature) of $\mathbb{S}_{\sqrt{\lambda}}^{n-1}$ is $\frac{n(n-1)}{\lambda}$, proposition 3.7.2 in [2] implies that

$$
\mathcal{O} p_{\hbar}^{\lambda}\left(l_{\lambda}^{2}\right)=\lambda \mathcal{O} p_{\hbar}^{\lambda}\left(g_{q}^{\lambda}\right)^{-1}=\lambda\left(-\hbar^{2} \Delta_{S}+\frac{n(n-1) \hbar^{2}}{4 \lambda}\right),
$$

where $\Delta_{S}$ is the Laplace-Beltrami operator on the sphere. It is well known that $-\hbar^{2} \lambda \Delta_{S}=L_{\lambda}^{2}$ and this finishes the proof.

It is well known that the principal symbol of the LaplaceBeltrami operator on any complete Riemannian manifold is $\left(g_{q}^{\lambda}\right)^{-1}(p, p)$ [24]. So our previous theorem comes to show that the well known relation between $L^{2}$ and the Laplace-Beltrami operator on the sphere also holds true classically.

Also notice that all the quantization the authors know for the cotangent bundle of a Riemannian manifold sent $\left(g_{q}^{\lambda}\right)^{-1}(p, p)$ to the Laplace-Beltrami operator plus some constant times the Ricci scalar. It seems that this was first noticed by Pauli [25]. So, the fact that $\mathcal{O} p_{\hbar}\left(l^{2}\right) \neq L^{2}$ turns to be natural. However, there is no agreement about what constant should multiply the Ricci scalar. In physics literature, many different constants have been proposed, as it was noticed in [26] [some further development can be found in [27]], where a proposal for such constant followed some general interesting ideas concerning the case when the Riemannian manifold is a hypersurface. Our result follows Weyl quantization and is based on the idea that the angular momenta coordinates on the cotangent bundle of the sphere should play the role of momenta coordinates on the flat Euclidean phase space.

\section{SPECTRAL ANALYSIS AND CQR}

In this section we will show that the first part of the spectral analysis of $L^{2}$ can be generalized to any operator of the form $\mathcal{O} p_{\hbar}(f)$, where $f$ is a constant of motion on which we have CQR and some extra symmetry is preserved. Any function of angular momenta preserves that symmetry. Let us introduce first some notation.

Let $D_{\lambda}: \mathbb{S}_{\sqrt{\lambda_{0}}}^{n-1} \rightarrow \mathbb{S}_{\sqrt{\lambda_{0} \lambda}}^{n-1}$ be the diffeomorphism given by $D_{\lambda}(z)=\sqrt{\lambda} z$ and $s_{\lambda}: T^{*} \mathbb{S}_{\sqrt{\lambda_{0}}}^{n-1} \rightarrow T^{*} \mathbb{S}_{\sqrt{\lambda_{0} \lambda}}^{n-1}$ the symplectomorphism given by

$$
s_{\lambda}(q, p)=\left(D_{\lambda}(q),\left(D_{\lambda}^{*}\right)^{-1}(p)\right)=\left(\sqrt{\lambda} q, \frac{1}{\sqrt{\lambda}} p\right) .
$$

If $\lambda_{0}=1$, then $s_{\lambda}$ is the classical counterpart of the unitary operator $S_{\lambda}$.

Notice that $D_{\lambda}$ (respectively $s_{\lambda}$ ) is the restriction of a map defined on $\mathbb{R}^{n}$ (respectively $T^{*} \mathbb{R}^{n}=\mathbb{R}^{2 n}$ ) given by the same expression, and they define a multiplicative one parameter group 
of diffeomorphisms (respectively symplectomorphism), i.e. $D_{1}=$ $I d$ and $D_{\lambda_{1} \cdot \lambda_{2}}=D_{\lambda_{1}} D_{\lambda_{2}}$ (respectively $s_{1}=I d$ and $s_{\lambda_{1} \cdot \lambda_{2}}=s_{\lambda_{1}} s_{\lambda_{2}}$. In fact, up to composing with the exponential, $D_{\lambda}$ correspond to the (additive) one parameter group of the gradient $\nabla \varphi$, where $\varphi(x)=\|x\|^{2}$. Therefore $s_{\lambda}$, after composing with the exponential, coincides with the Hamiltonian one parameter group of $J_{\nabla \varphi}$.

THEOREM 6. Let $f$ be an admissable constant of motion such that $f\left(t x, t^{-1} \xi\right)=f(x, \xi)$, for any $t>0$. Then

$$
f_{\lambda} \circ s_{\lambda}=f_{1}
$$

and

$$
\mathcal{O} p_{\hbar}^{\lambda}\left(f_{\lambda}\right)=S_{\lambda}^{*} \mathcal{O} p_{\hbar}^{1}\left(f_{1}\right) S_{\lambda}
$$

Proof: The first equality follows directly from the condition $f\left(t x, \frac{1}{t} \xi\right)=f(x, \xi)$ and the definition of $f_{\lambda}$. Using the expression for $\mathcal{O} p_{\hbar}^{\lambda}$ given in Belmonte [16], section 5, we obtain

$$
\begin{aligned}
& \mathcal{O} p_{\hbar}^{1}\left(f_{1}\right) S_{\lambda} u(z) \\
& =\hbar^{1-n} \lambda^{\frac{n-1}{4}} \int_{\mathbb{S}^{n-1}-\{-z\}} \hat{f}_{1}\left(\frac{(z+w)}{\|z+w\|}, \frac{\theta}{\hbar} \frac{(z-w)}{\|z-w\|}\right) u(\sqrt{\lambda} w) d \mu(w) \\
& =\hbar^{1-n} \lambda^{\frac{1-n}{4}} \int_{\mathbb{S}_{\sqrt{\lambda}}^{n-1}-\{-\sqrt{\lambda} z\}} \hat{f}_{1}\left(\frac{\sqrt{\lambda} z+w}{\|\sqrt{\lambda} z+w\|}, \frac{\theta}{\hbar} \frac{(\sqrt{\lambda} z-w)}{\|\sqrt{\lambda} z-w\|}\right) u(w) d \mu(w)
\end{aligned}
$$

Notice that the Fourier transform $\hat{f}_{1}$ is computed in the cotangent plane of $\mathbb{S}^{n-1}$ at the point $q=\frac{\sqrt{\lambda} z+w}{\|\sqrt{\lambda} z+w\|}$. Let $v=\frac{\theta}{\hbar} \frac{(\sqrt{\lambda} z-w)}{\|\sqrt{\lambda} z-w\|}$. Then

$$
\begin{aligned}
\hat{f}_{1}(q, v) & =\int_{T_{q}^{*} \mathbb{S}^{n-1}} f_{1}(q, p) e^{i p \cdot v} d \mu_{q}^{*}(p) \\
& =\int_{T_{q}^{*} \mathbb{S}^{n-1}} f_{1}(q, p) e^{i \lambda^{-\frac{1}{2}} p \cdot \lambda^{\frac{1}{2}} v} d \mu_{q}^{*}(p) \\
& =\lambda^{\frac{n-1}{2}} \int_{T_{\sqrt{\lambda} q}^{*} \mathbb{S}^{n-1} \sqrt{\lambda}}\left(f_{1} \circ s_{\lambda^{-1}}\right)(\sqrt{\lambda} q, p) e^{i p \cdot \sqrt{\lambda} v} d \mu_{q}^{*}(p) .
\end{aligned}
$$

Therefore

$$
\begin{aligned}
& \mathcal{O} p_{\hbar}^{1}\left(f_{1}\right) S_{\lambda} u(z) \\
& =\hbar^{1-n} \lambda^{\frac{n-1}{4}} \int_{\mathbb{S}_{\sqrt{\lambda}}^{n-1}-\{-\sqrt{\lambda} z\}} \hat{f}_{\lambda}\left(\frac{\sqrt{\lambda}(\sqrt{\lambda} z+w)}{\|\sqrt{\lambda} z+w\|}, \frac{\sqrt{\lambda} \theta}{\hbar} \frac{(\sqrt{\lambda} z-w)}{\|\sqrt{\lambda} z-w\|}\right) u(w) d \mu(w) \\
& =S_{\lambda} \mathcal{O} p_{\hbar}^{\lambda}\left(f_{\lambda}\right) u(z)
\end{aligned}
$$

COROLlary 7. Let $f$ be an admissable constant of motion such that $f\left(t x, t^{-1} \xi\right)=f(x, \xi)$, for any $t>0$.

1. Then, for any $\lambda \in[0, \infty)$, we have that

$$
\operatorname{sp}\left(\mathcal{O} p_{\hbar}^{d}(f)\right)=\operatorname{sp}\left(\mathcal{O} p_{\hbar}^{\lambda}\left(f_{\lambda}\right)\right)
$$

2. If $\mathcal{O} p_{\hbar}^{\lambda}\left(f_{\lambda}\right)$ is bounded, for some $\lambda \in[0, \infty)$. Then $\mathcal{O} p^{d}(f)$ is bounded and

$$
\left\|\mathcal{O} p_{\hbar}^{d}(f)\right\|=\left\|\mathcal{O} p_{\hbar}^{\lambda}\left(f_{\lambda}\right)\right\| .
$$

Proof: Both identities are direct consequences of the previous theorem and the equalities (5) and (6).

COROLLARY 8. Iff is a constant of motion such that $f\left(t x, t^{-1} \xi\right)=$ $f(x, \xi)$, for any $t>0$, and we have CQR on $f$, then for any $\lambda \in[0, \infty)$ we have that

$$
\operatorname{sp}\left(\mathcal{O} p_{\hbar}(f)\right)=\operatorname{sp}\left(\mathcal{O} p_{\hbar}^{\lambda}\left(f_{\lambda}\right)\right)
$$

\section{Remark:}

Notice that since $l_{i j}\left(t x, t^{-1} \xi\right)=l_{i j}(x, \xi)$, every function of angular momenta satisfies that condition and therefore we can apply our results on them. However, not every classical constant of motion satisfies that condition, for instance $h_{0}$ itself does not. The constants of motion satisfying $f\left(t x, t^{-1} \xi\right)=f(x, \xi)$ are completely determined by their value on the set $\{(x, \xi) \in$ $\left.\mathbb{R}^{2 n} /\|\xi\|=1 ;\langle x, \xi\rangle=0\right\}$. The fact that not every $f$ is such that $f\left(t x, t^{-1} \xi\right)=f(x, \xi)$ is the classical counterpart of the fact that not every quantum constant of motion is completely determined by its restriction to one sphere, as we discussed in subsection 3.2.

The last corollary 8 gives further reasons to look for a proof to our conjecture, at least for functions of angular momenta.

\section{DISCUSSION AND FUTURE WORK}

The technique used to prove theorem 4 and corollary 5 are interesting on its own right and lead to some further questions; we shall briefly explain why. Let $\mathfrak{g}$ be a Lie algebra and let $U(\mathfrak{g})$ be the corresponding universal enveloping algebra. In general, Casimir elements by definition belong to the center of $U(\mathfrak{g})$. Using the universal property of $U(\mathfrak{g})$, we can represent it on $C^{*}\left(\mathfrak{g}^{*}\right)$. In our case, we were working with the quadratic Casimir, but one can consider any symmetric homogeneous polynomial to build Casimir elements. On the other hand, if $\mathfrak{g}$ is the Lie algebra of a Lie group $G$, we can identify $U(\mathfrak{g})$ with the algebra of leftinvariant differential operators on $G$; it turns out that the operator corresponding to the quadratic Casimir is precisely the LaplaceBeltrami operator on $G$ (endowed with its canonical Riemannian structure). Moreover, if $G$ acts on some Riemannian manifold $M$ and such action satisfies certain condition, then the quadratic Casimir is represented as the Laplace-Beltrami operator on $M$ (this seems to be the case when $M$ is a $G$-homogeneous space; for instance $M=\mathbb{S}^{n-1}$ and $\left.G=S O(n)\right)$. We can also represent $U(\mathfrak{g})$ on $C^{\infty}\left(T^{*} M\right)$ as well. So, our theorem and the proof of the corollary show that Weyl-Landsman quantization factorizes both representations up to some lower degree term, at least for the quadratic Casimir and $\mathfrak{g}=\mathfrak{s o}(n)$. One should wonder if this holds true for any element of $U(\mathfrak{g})$, in particular for any Casimir element. The latter would be already interesting for quartic Casimirs, since they are related with the stress-energy tensor. Heuristically, such result would mean that the representation on $C^{\infty}\left(\mathfrak{g}^{*}\right)$ (and therefore on $C^{\infty}\left(T^{*} M\right)$ ) can be interpreted as a 
dequantization of $U(\mathfrak{g})$. For details see Barut and Raczka [28] or Fuchs and Schweigert [29].

Coming back to our context, recall that our main purpose is to understand the decomposition of the operators of the form $\mathcal{O} p_{\hbar}(f)$ through the diagonalization of the Laplace operator on $\mathbb{R}^{n}$, where $f$ is a classical constant of motion for $h(x, \xi)=\|\xi\|^{2}$. Also recall that functions of angular momenta are examples of such constants of motion. The latter claim is another way to say that every function of the form $J^{*} \varphi$ is a constant of motion, where $J: \mathbb{R}^{2 n} \rightarrow \mathfrak{s o}^{*}(n)$ is the canonical moment map and $\varphi \in C^{\infty}\left(\mathfrak{s o}^{*}(n)\right)$. Notice that $\left[J^{*} \varphi\right]_{\lambda}=J_{\lambda}^{*} \varphi$. Therefore, studying the problem described in the previous paragraph would lead to some further interesting examples where we might have that our conjecture $\left[\mathcal{O} p_{\hbar}(f)\right]_{\lambda}=\mathcal{O} p_{\hbar}^{\lambda}\left(f_{\lambda}\right)$ holds.

From a physically and a bit more speculative perspective, we would like to state that it is remarkable that the method introduced in this paper leaves aside the requirement of mapping position and momentum coordinates, and focuses on the mapping of COM themselves. Indeed, recall that the state of a quantum system is not determined by the position and momentum coordinates, but by a complete set of commuting observables (CSCO). Since CSCO usually includes

\section{REFERENCES}

1. Groenewold HJ. On the principles of elementary quantum mechanics. Physica (1946) 12:405-60.

2. Landsman NP. Mathematical Topics Between Classical and Quantum Mechanics, Springer Monographs in Mathematics. New York, NY: SpringerVerlag (1998).

3. Woodhouse NMJ. Geometric Quantization.Oxford: Oxford University Press (1997).

4. Steven W. The Quantum Theory of Fields, Vol. 2. Cambridge: Cambridge University Press (1995).

5. Landsman NP. Between classical and quantum. Handb Philos Sci. (2006) 417-553.

6. Rieffel MA. Deformation quantization of Heisenberg Manifolds. Comm Math Phys. (1989) 122:531-62.

7. Kontsevich M. Deformation quantization of Poisson manifolds. Lett Math Phys. (2003) 66:157-216.

8. Bayen F, Flato M, Fronsdal C, Lichnerowicz A, Sternheimer D. Deformation theory and quantization, Part I. Lett. Math. Phys. (1977) 1: 521-30.

9. Bayen F, Flato M, Fronsdal C, Lichnerowicz A, Sternheimer D. Deformation theory and quantization, Part II and III. Ann. Phys. (1978) 111:111-151.

10. Souriau J-M. Quantification géométrique. Commun Math Phys. (1966) 1:374-98.

11. van Hove L. Sur Certaines Représentations Unitaires d'un Groupe Infini de Transformations. Ph.D. Thesis, Bruxelles University (1951).

12. Folland GB. Harmonic Analysis in Phase Space. Annals of Mathematics Studies, Vol. 122. Princeton, NJ: Princeton University Press (1989).

13. Fine A. Hidden variables, joint probability, and the Bell inequalities. Phys Rev Lett. (1982) 48:291.

14. Goldstein H. Classical Mechanics. London: Pearson Education India (2011).

15. Miura RM, Gardner CS, Kruskal MD. Kortewegde Vries equation and generalizations. II. Existence of conservation laws and constants of motion. J Math Phys. (1968) 9:1204-9.

16. Belmonte F. Quantization of Constants of Motion. arXiv:1505.07250 (2018).

17. Abraham R, Marsden E. Foundations of Mechanics, 2nd Edn. Reading, MA: Benjamin/Cummings Publishing Co. (1978).

18. Birman MS, Solomyak MZ. Spectral Theory of Selfadjoint Operators in Hilbert Spaces. Translated from the 1980 Rusian Original by S. Khrushchev and the Hamiltonian, then the observables in CSCO are COM. Therefore a quantization that preserves COM will necessarily map a classical system into a quantum system where the description of the states can be directly determined from the quantized operators. In order to develop a decomposable Weyl calculus where the latter is possible, one should apply recursively the decomposable quantization calculus for each operator in the CSCO. If that is possible, we would obtain an intuitively more appropriate quantization than the regular quantization that demands the quantization of position and momentum coordinates.

\section{AUTHOR CONTRIBUTIONS}

The ground of the CQR mathematical framework presented in this article was developed by FB, while for the application of CQR to the angular momentum and the discussion around it both authors contributed equally.

\section{FUNDING}

TV has been supported by the Fondecyt postdoctoral project 3170122 .

V. Peller. Mathematics and its Applications (Soviet Series). Dordrecht: Reidel Publishing Co. (1987).

19. Dixmier J. Von Neumann Algebras. Jellett F. Translated from Les algèbres d'operators dans l'espace hilbertien(algèbres de Von Neumann), Vol. 27. NorthHolland Mathematical Library (1969).

20. Jensen A. Time-delay in Potential scattering theory. Commun Math Phys. (1981) 82:435-56.

21. Pelayo A, Vu Ngoc S. Spectral limits of semiclassical commuting selfadjoint operators. A mathematical tribute to Professor José María Montesinos Amilibia. Dep. Geom. Topol. Fac. Cien. Mat. UCM, Madrid (2016) 527-46.

22. Pelayo A, Polterovich L, Vu Ngoc S. Semiclassical quantization and spectral limits of $\hbar$-Pseudodifferential and Berezin-Toeplitz Operators. Proc Lond Math Soc. (2014) 109:676-96. doi: 10.1112/plms/pdu015

23. Griffiths DJ. Introduction to Quantum Mechanics. Cambridge: Cambridge University Press (2016).

24. Shubin MA. Pseudodifferential Operators and Spectral Theory, Springer Series in Soviet Mathematics. Translated by Andersson SI. Berlin: Springer (1987).

25. Pauli W. Ausgewahlte Kapital aus der Feldquantisierung, E.T.H. Zurich Lecture Notes 1950-51. In: Enz CP editor. Pauli Lectures on Physics, Vol. 6: Selected Topics in Field Quantization. Cambridge: MIT Press (1973).

26. Lian D, Hu L, Liu Q. Geometric potential and dirac quantization. Annalen der Physik (2018) 530:1700415. doi: 10.1002/andp.201700415

27. Liu QH. Geometric momentum for a particle constrained on a curved hypersurface. J Math Phys. 2013 54:122113. doi: 10.1063/1.4854075

28. Barut AO, Raczka, R. Theory of group representations and applications, 2nd Edn. Singapore: World Scientific Publishing Co. (1986).

29. Fuchs J, Schweigert C. Symmetries, Lie Algebras and Representations. A Graduate Course for Physicists. Cambridge Monographs on Mathematical Physics. Cambridge: Cambridge University Press (1997).

Conflict of Interest Statement: The authors declare that the research was conducted in the absence of any commercial or financial relationships that could be construed as a potential conflict of interest.

Copyright (c) 2018 Belmonte and Veloz. This is an open-access article distributed under the terms of the Creative Commons Attribution License (CC BY). The use, distribution or reproduction in other forums is permitted, provided the original author(s) and the copyright owner(s) are credited and that the original publication in this journal is cited, in accordance with accepted academic practice. No use, distribution or reproduction is permitted which does not comply with these terms. 